\title{
The comparison of perioperative outcomes of robot-assisted and open partial nephrectomy: a systematic review and meta-analysis
}

Zhonghua Shen ${ }^{1,2 \dagger}$, Linguo Xie ${ }^{1,2+}$, Wanqin Xie ${ }^{3 \dagger}$, Hailong Hu ${ }^{1,2}$, Tao Chen ${ }^{1,2}$, Chen Xing ${ }^{1,2}$, Xiaoteng Liu', Hao $\mathrm{Xu}^{1,2}, \mathrm{Yu}$ Zhang ${ }^{1,2}$, Zhouliang $\mathrm{Wu}^{1,2}$, Dawei $\operatorname{Tian}^{1,2^{*}}$ and Changli $\mathrm{Wu}^{1,2^{*}}$

\begin{abstract}
Background: Robot-assisted partial nephrectomy (RAPN) has been widely used worldwide, to determine whether RAPN is a safe and effective alternative to open partial nephrectomy (OPN) via the comparison of RANP and OPN.

Methods: A comprehensive literature search was performed within the databases including PubMed, Cochrane Library, and Embase updated on 30 September 2015. Summary data with their corresponding $95 \%$ confidence intervals (Cls) were calculated using a random effects or fixed effects model. Heterogeneity and publication bias were also evaluated.

Results: A total of 16 comparative studies including 3024 cases were used for this meta-analysis. There are no significant differences in the demographic characteristic between the two groups, but the age was lower and the tumor size was smaller for the RAPN group. RAPN had a longer operative time and warm ischemia time but which showed less estimated blood loss, hospital stay, and perioperative complications. No differences existed in the margin status, the change of glomerular filtration rate, transfusion rate, and conversion rate between the two groups. There was no significant publication bias.
\end{abstract}

Conclusions: RAPN offered a lower rate of perioperative complications, less estimated blood loss, and shorter length of hospital stay than OPN, suggesting that RAPN can be an effective alternative to OPN. Well-designed prospective randomized controlled trials will be helpful in validating our findings.

Keywords: Robot-assisted partial nephrectomy, Open partial nephrectomy, Renal tumor, Meta-analysis

Abbreviations: RAPN, Robot-assisted partial nephrectomy; OPN, Open partial nephrectomy; BMl, Body mass index; WMD, Weighted mean difference; OR, Odds ratio; Cl, Confidence interval; EAU, European Association of Urology; LPN, Laparoscopic partial nephrectomy; RCTs, Randomized controlled trials; OT, Operating time; WIT, Warm ischemia time; HS, Hospital stay; PSM, Positive surgical margins; eGFR, Glomerular filtration rate; NOS, The Newcastle-Ottawa Scale; $\mathrm{R}$, Retrospective; RP, Retrospective analysis, prospective data collecting; PN, Prospective non-randomized design; EBL, Estimated blood loss

\footnotetext{
* Correspondence: daweitian@163.com; wujygc2003@163.com

${ }^{\dagger}$ Equal contributors

'Department of Urology, The Second Hospital of Tianjin Medical University,

Pingjiang Road 23Hexi District, Tianjin 300211, China

Full list of author information is available at the end of the article
} 


\section{Background}

Renal tumor has become a significant threat to the public health, with a high incidence rate of $12.6 / 10,000$ and 6.7/10,000 for men in the developed and less developed regions, respectively [1]. Partial nephrectomy and radical nephrectomy are the two surgical options to deal with renal tumor. Because partial nephrectomy may have less renal function impairment, better overall survival, and equivalent oncological survival compared to radical nephrectomy, the European Association of Urology recommended partial nephrectomy, when feasible, as a gold standard treatment for patients presenting with small renal carcinoma [2].

There are three different ways to perform partial nephrectomy, namely the open partial nephrectomy $(\mathrm{OPN})$, laparoscopic partial nephrectomy (LPN), and robot-assisted partial nephrectomy (RAPN). Among these available nephron-sparing surgery options, OPN is the most extensively studied and has been demonstrated with comparable oncological efficacy, less incidence of chronic kidney disease within a 10-year follow-up, less cardiovascular morbidity, and overall mortality compared to radical nephrectomy $[3,4]$. LPN has been shown to offer better cosmetic results, less postoperative pain, shorter length of hospital stay, and faster postoperative recovery than OPN, but the steep learning curve leads to its limited diffusion in the high-volume reference centers and application to the small and less complex tumors [5]. Although a few studies have compared the perioperative outcomes of OPN and RAPN, their results are inconsistent. Han et al. [6] and Alemozaffar et al. [7] found that RAPN caused longer operative time than OPN, whereas other studies [8-10] showed the opposite finding. Regarding the complication rate, the comparison between RAPN and OPN in two studies $[11,12]$ also yielded with conflicting results.

Based on these observations, we believe there is a necessity for a systematic review to compare the perioperative outcomes of RAPN and OPN with the most updated data and draw a more accurate conclusion.

\section{Methods}

\section{Publication search}

The systematic review followed the Cochrane review guidelines. A comprehensive literature search using the combinations of key words "open," "robotic/robotassisted," and "partial nephrectomy" was done within the electronic databases PubMed, Cochrane Library, and Embase updated on 30 September 2015. The publications in English rather than other languages were collected. The computer search was supplemented with manual searches within the reference lists of all retrieved studies, review articles, and conference abstracts. Two authors (Chen Tao and $\mathrm{Hu}$ Hailong) reviewed the titles and abstracts of all items returned by the search engine to assess their relevance to this meta-analysis independently. When the two authors could not make an agreement on certain items, Xie Linguo served as a third reviewer to look into the full text and make a decision that whether the publication is qualified for this study.

\section{Inclusion and exclusion criteria}

All available randomized controlled trials (RCTs) and retrospective comparative studies (cohort or casecontrol studies) that compared RAPN with OPN were included. Studies of comparison of RAPN, LPN, and OPN were also included as long as the data for RAPN and OPN could be extracted and had at least one of the outcomes mentioned in the paper. Editorials, review articles, and animal experimental studies were excluded. When multiple reports described the same population, the most recent or complete report was used.

\section{Data extraction}

Data associated with the included studies were extracted and summarized by two authors (Chen Tao and XieLinguo) independently. A senior author $(\mathrm{Hu}$ Hailong) was responsible for resolving disagreements pertaining to data extraction if there were any. The extracted information contained the demographic data (age, gender, body mass index, tumor size, and the location of tumor), the information about the source of controls, study design, and sample size of the study population, and the perioperative outcomes including operating time (OT), warm ischemia time (WIT), blood loss, length of hospital stay (HS), positive surgical margins (PSM) rate, change of glomerular filtration rate (eGFR), perioperative complications rate, transfusion rate, and conversion rate (RPN converted to laparoscopic or open partial nephrectomy, RPN or OPN converted to radical nephrectomy). The day of follow-up, the number of tumor recurrence, metastasis, and death were also extracted.

\section{Statistical analysis and quality assessment}

The random effects model was used when significant heterogeneity existed among the included studies as assessed by the inconsistency index $\left(I^{2}>50 \%\right)$ and the chi-square test with significance heterogeneity $(p<0.10)$; otherwise, the fixed effects model was used [13]. The level of evidence of the included studies was rated according to the criteria provided by the Centre for Evidence-Based Medicine in Oxford, UK (CEBM home page, available online http:// www.cebm.net/index.aspx?o=1025, accessed on September 5, 2014). The modified Newcastle-Ottawa scale was used to assess the quality of the retrospective study [14] (The Newcastle-Ottawa Scale (NOS) for assessing the quality of non-randomized studies in meta-analyses. http://www.ohri.ca/programs/clinical_epidemiology/oxford.asp, accessed 
on September 15, 2014). This evaluation system consists of three factors: assessment of outcome, comparability of the study groups, and patient selection. A score ranging from 0 to 9 (represented by 0 to 9 stars) was provided as the result of the assessment for each study, except for RCTs (Table 1). The studies which scored by 6 or more stars were considered to be of high quality. All the metaanalyses were performed using Review Manager 5.2 (Cochrane Collaboration, Oxford, UK). Weighted mean difference (WMD) and odds ratio (OR) were used to compare continuous and dichotomous variables, respectively. All results were reported with $95 \%$ CIs. All reported $p$ values were two sides, the value of $p<0.05$ was considered to indicate statistical significance, and publication bias was assessed by Funnel plots. The Clavien-Dindo score system was used to stratified postoperative complication for subgroup analysis [15].

\section{Results}

\section{Literature search and characteristics of the included} studies

The results for each step of the literature search are shown in Fig. 1. Eventually, a total of 16 publications fulfilled the predefined inclusion criteria, including 15 full-text articles [6-12, 16-23] and 1 conference abstract [24]. Two of the 13 studies compared the outcomes of patient groups stratified by RENAL score $[12,20]$. Thus, in total, there were 18 independent study populations included in this metaanalysis. Of the 16 studies, 4 studies conducted the comparison of perioperative outcomes of RAPN, LPN, and OPN $[6,7,18,21], 12$ studies documented their single center's experience with RAPN versus OPN $[6-10,12,16$, $18,19,21-23], 3$ studies [11, 17, 20] involved the multicenter collaboration, and 10 studies [6, 7, 11, 12, 17-20, 22, 23] matched the two approach with renal tumor nephrometry score (RENAL or PADUA). Among the included studies, there was 2 prospective nonrandomized comparative study (level of evidence: II) [8, 11], 3 studies retrospective analysis but prospective data collecting (level of evidence: III) $[6,12,16], 9$ retrospective studies compared contemporary of patients (level of evidence: III) [7, 10, 17-23], 1 retrospective study used a historical series as the control (level of evidence: IV) [9], and 1 retrospective study but a conference abstract (level of evidence: IV) [24].

Our literature search found no randomized and blinded studies available for this meta-analysis. Seven studies $[8,10,12,16,18,20,23]$ declared the duration of follow-up for both groups. Table 1 summarizes the characteristics of the included studies.

\section{Demographic characteristics of the study populations}

This meta-analysis involved 3024 cases (1103 cases for RAPN and 1921 cases for OPN) (Table 2). There are no significant differences in the gender, patients of benign: malignant, and body mass index (BMI) of patients between RAPN and OPN, but the age was lower (WMD, -1.52 years; $95 \% \mathrm{CI},-2.53$ to $-0.51 ; p=0.003$ ) and the tumor size was smaller for RAPN group (WMD, $-0.46 \mathrm{~cm} ; 95 \% \mathrm{CI},-0.66$ to $-0.26 ; p<0.01$ ).

\section{Comparison of perioperative outcomes of RAPN and OPN}

Table 3 summarizes the perioperative outcomes of RAPN and OPN.

The operative time was statistically shorter in the OPN group (WMD, $27.79 \mathrm{~min}$; $95 \% \mathrm{CI}, 4.51$ to $51.07 ; p=0.02$ ) (Fig. 2a), but the estimated blood loss (WMD, $-105.57 \mathrm{ml}$; $95 \%$ CI, -160.78 to -50.36 ; $p=0.0002$ ) (Fig. 2b) was less in the RAPN group, and a significantly shorter postoperative hospital stay (WMD, -2.06 day; $95 \% \mathrm{CI},-2.62$ to $-1.51 ; p$ $<0.001$ ) (Fig. 2c) was found in the RAPN group. Eight studies $[8,10,17,19-21,23,24]$ examined the perioperative transfusion, and we found that there was no significant difference in transfusion rate between RAPN and OPN (OR, 0.86; $95 \%$ CI, 0.56 to 1.32; $p=0.50$ ) (Fig. 2d). Seven studies [9-12, 18, 21, 24] made the comparison of intraoperative conversion rate of RAPN and OPN; as a result, we found no difference in conversion rate between RAPN and OPN (OR, 0.91; $95 \%$ CI, 0.38 to 2.14; $p=0.83$ ) (Fig. 2e). Thirteen of the 16 included studies for this meta-analysis examined the margin status of surgical specimens [8-12, 16-21, 23, 24], no significant difference was observed regarding the positive margin rate between RAPN and OPN based on the data from the 11 studies involving 2220 cases (OR, 0.93; $95 \% \mathrm{CI}, 0.57$ to $1.52 ; p=0.78$ ) (Fig. 2f). The overall warm ischemia time of RAPN was significantly longer than that of OPN (WMD, $2.18 \mathrm{~min}$; $95 \% \mathrm{CI}, 0.49$ to $3.87 ; p=0.01$ ) as supported by the pooled data from 10 studies $[6,8,9,11,12,19,20,23,24]$ (Fig. 2g). There was no difference in the change of eGFR between RAPN and OPN (WMD, $-0.56 \mathrm{ml} / \mathrm{min}$ per $1.73 \mathrm{~m}^{2}$; $95 \%$ CI, -2.35 to $1.23 ; p=0.54$ ) (Fig. $2 \mathrm{~h}$ ).

\section{Comparison of complications of RAPN and OPN}

Table 4 summarizes the complications of the two groups.

The overall complication rate of RAPN was significantly lower than that of OPN (OR, 0.64; $95 \% \mathrm{CI}$, 0.51 to $0.79 ; p<0.001$ ) by the pooled data from 15 studies $[6,8,10-12,16-24]$ (Fig. 2i). Intraoperative complication rate was available for 8 studies [8-11, 17-20], and no significant difference existed between the two groups (OR, 0.86; 95 \% CI, 0.42 to 1.76; $p=0.68$ ) (Fig. 2j). Postoperative complication rate was mentioned in 13 studies $[6,8-11,16-20,22-24]$, and patients from the 11 studies $[6,9-11,16-18,20,22-24]$ were further divided into the minor (Clavien classification 1-2) complication subgroup and major (Clavien classification 3-5) complication subgroup. The pooled data favored RAPN for lower rates of overall postoperative complication (OR, 
Table 1 Characteristics of the included studies

\begin{tabular}{|c|c|c|c|c|c|c|}
\hline Study & Level of evidence & Design & Matching $^{a}$ & Follow-up (month) ${ }^{f}$ RAPN/OPN & No. of centers & Quality score $^{g}$ \\
\hline Wu et al. [10] & $\mathrm{lllb}$ & $R$ & $1,2,3,4,6,8$ & $12(6-24) / 12(6-24)$ & Single & 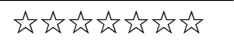 \\
\hline Vittori et al. [11] & $\|$ & PN & $1,2,3,4,7,8$ & Perioperative & Multicenter & 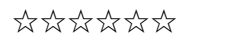 \\
\hline Masson-Lecomte et al. [16] & Illb & $\mathrm{RP}$ & $1,2,3,4,6,8$ & $19(6-30) / 32(12-40)$ & Single & 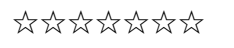 \\
\hline Ficarra et al. [17] & $\mathrm{lllb}$ & $\mathrm{R}$ & $1,2,5,6,7,8$ & Perioperative & Multicenter & 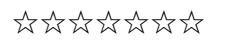 \\
\hline Kim et al. [24] & IV & $\mathrm{R}$ & $1,2,3,4,5,6$ & NA/NA & NA & 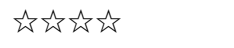 \\
\hline Lucas et al. [18] & Illb & $\mathrm{R}$ & $1,2,3,6,7,8,9$ & $9.4 \pm 7.6 / 21.1 \pm 18.8$ & Single & 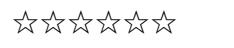 \\
\hline Han et al. [6] & Illb & $\mathrm{RP}$ & $1,2,4,5,6,7,8$ & Perioperative & Single & 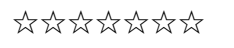 \\
\hline Oh et al. [19] & Illb & $\mathrm{R}$ & $1,2,3,4,5,6,7,8,9$ & Perioperative & Single & 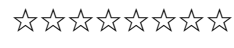 \\
\hline Lee et al. [9] & IV & $\mathrm{R}$ & $1,2,5,6,8$ & NA/NA & Single & 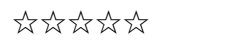 \\
\hline Alemozaffar et al. [7] & $\mathrm{lllb}$ & $\mathrm{R}$ & $2,3,4,5,6,7$ & Perioperative & Single & 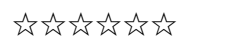 \\
\hline Simhan et al. [12] & Illb & $\mathrm{RP}$ & $1,2,3,4,6,7$ & $17.1 \pm 9.2 / 23.9 \pm 20.5^{b} ; 21.3 \pm 13.3 / 19.8 \pm 11.1^{c}$ & Single & 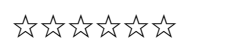 \\
\hline Zargar et al. [20] & $\| l l b$ & $\mathrm{R}$ & $1,2,3,5,6,7$ & $7.8(18.7) / 14(14.5)^{\mathrm{d}} ; 4(18) / 19.6(29.7)^{\mathrm{e}}$ & Multicenter & 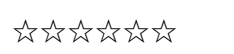 \\
\hline Boylu et al. [8] & $\|$ & PN & $1,2,3,5,6,8$ & $33 \pm 13 / 37 \pm 20$ & Single & 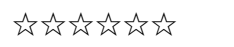 \\
\hline Mano et al. [22] & $\| l l b$ & $\mathrm{R}$ & $1,2,3,4,5,6,7$ & Perioperative & Single & 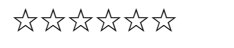 \\
\hline Miyake et al. [23] & Illb & $\mathrm{R}$ & $1,2,3,4,5,6,7,8$ & $1 / 1$ & Single & 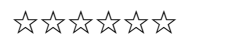 \\
\hline Webb et al. [21] & $\| l l b$ & $\mathrm{R}$ & $1,2,5,6,8$ & NA/NA & Single & 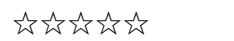 \\
\hline
\end{tabular}

$R$ retrospective, $R P$ retrospective analysis, prospective data collecting, $P N$ prospective non-randomized design, $N A$ not available

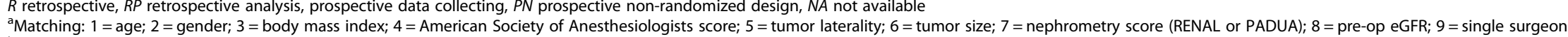
a Matching: 1 = age; 2 = gender; 3 = body
${ }^{\mathrm{b}}$ Moderate nephrometry group (NS 7-9)

'High nephrometry group (NS 10-12)

dSimple tumors (RENAL score 4-8)

${ }^{\text {e}}$ Complex tumors (RENAL score 9-12)

"The follow-up time was reported in the form of "median (inter-quartile range)" or "mean \pm standard deviation" or not recorded

${ }^{9}$ Modified Newcastle-Ottawa Scale (NOS) 


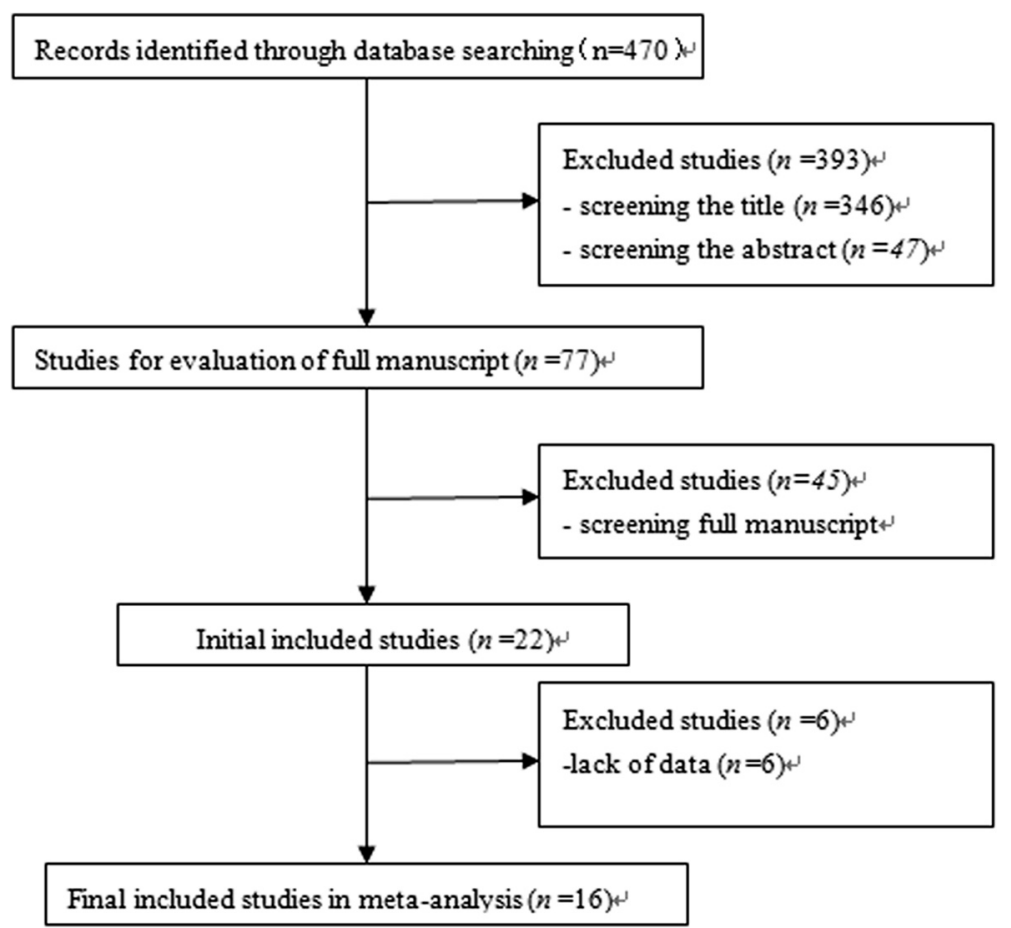

Fig. 1 Flow diagram of the comprehensive literature search

0.64; $95 \%$ CI, 0.51 to $0.79 ; p<0.001$ ) (Fig. $2 \mathrm{k}$ ), minor complication (OR, 0.62; $95 \% \mathrm{CI}, 0.46$ to $0.83 ; p=0.001$ ), and major complication (OR, 0.57; $95 \% \mathrm{CI}, 0.36$ to 0.91 ; $p=0.02$ ) compared to OPN.

\section{Comparison of postoperative efficacy of RAPN and OPN}

Eight studies [8-10, 12, 16, 18, 20, 21] compared the recurrence or metastasis rate of the RAPN with that of the OPN. Among the 8 studies, there were 13 recurrences, 1 metastasis, and 5 deaths in the OPN group, 756 patients were included, while there were 0 recurrence, 1 metastasis, and 2 deaths in the RAPN group including 380 patients. The ratio of tumor recurrence metastasis and death were 2.5 and $0.8 \%$ in the OPN and RAPN, respectively. However, the data were not pooled for meta-analysis due to the different lengths of follow-up period between the studies.

\section{Heterogeneity and publication bias analysis}

In this meta-analysis, the Q-test and the $I^{2}$ index were used to evaluate the heterogeneity across studies. As shown in Fig. 2, there was no heterogeneity among the Dichotomous variable, on the contrary, there was statistically significant heterogeneity among the continuous variables. Figure 3 shows that the funnel plots of the studies included in this meta-analysis reported perioperative complication rates. Almost all studies lie inside the $95 \%$ CIs (overall complication with $P=0.30$; intraoperative complication with $P=0.63$; minor complication with $P=0.58$; major complication with $P=0.07$ ), with an even distribution around the vertical, indicating no obvious reporting bias.

\section{Discussion}

LPN and RAPN have been increasingly accepted as minimally invasive nephron-sparing surgical modalities over the past two decades. Several studies $[25,26]$ have made the comparison and concluded that the surgical and oncologic outcomes provided by LPN are comparable to those by OPN. However, LPN requires proficient laparoscopic skills and the steep learning curve limits its wide application in partial nephrectomy. With the rapid advances in technology, RAPN has emerged as a new option to deal with small renal tumors, but whether PARN is safe and effective to deal with renal masses, we should compare it to OPN that is the matching standard of treatment and has the robustness of data regarding surgical and oncological results [27]. To make a comparison between RAPN and OPN, Wu et al. [10] performed a meta-analysis with the data extracted from 8 studies including 3418 patients (757 patients in the robotic group and 2661 patients in the open group); however, 2 of the 8 studies compared only the cost between RAPN and OPN [28, 29], and thus only 1118 patients (359 patients in the robotic group and 759 patients in the open group) accounted for the comparison in their study.

Here, we collected the data from 16 studies [6-8, 1012, 16-24] including 3024 cases (1103 cases for RAPN 
Table 2 Demographic and perioperative results of RAPN vs OPEN

\begin{tabular}{|c|c|c|c|c|c|c|}
\hline Study (OPN/RAPN) & Patients & Males & Age mean $\pm S D$ (year) & Benign: malignant & Tumor size mean \pm SD size $(\mathrm{cm})$ & $\mathrm{BMI}$ mean $\pm \mathrm{SD}\left(\mathrm{kg} / \mathrm{m}^{2}\right)$ \\
\hline Wu et al. [10] & $94 / 51$ & $62 / 35$ & NA/NA & NA:NA/NA:NA & NA/NA & NA/NA \\
\hline Vittori et al. [11] & 198/105 & $123 / 69$ & $63.8 \pm 12.4 / 62.3 \pm 11.6$ & 42:156/14:91 & $3.5 \pm 1.8 / 2.8 \pm 1.5$ & NA/NA \\
\hline Masson-Lecomte et al. [16] & $58 / 42$ & $40 / 22$ & $60.8 \pm 11.2 / 61.7 \pm 10.9$ & 8:33/6:52 & $3.1 \pm 1.2 / 2.8 \pm 1.4$ & $26.5 \pm 5.6 / 26.9 \pm 4.2$ \\
\hline Ficarra et al. [17] & 200/200 & $131 / 121$ & $62.4 \pm 11.8 / 62.4 \pm 10.6$ & $N A: N A / N A: N A$ & NA/NA & NA/NA/ \\
\hline Kim et al. [24] & $83 / 67$ & $56 / 48$ & $56.2 \pm 14.7 / 51.5 \pm 11.9$ & NA:NA/NA:NA & $2.6 \pm 1.7 / 2.3 \pm 1.1$ & $24.8 \pm 3.3 / 24.2 \pm 3.0$ \\
\hline Lucas et al. [18] & $54 / 27$ & $38 / 19$ & NA/NA & 10:44/10:17 & NA/NA & NA/NA \\
\hline Han et al. [6] & $354 / 147$ & $270 / 108$ & $55.3 \pm 12.4 / 52.5 \pm 11.9$ & NA:NA/NA:NA & $2.80 \pm 1.35 / 2.58 \pm 1.13$ & $24.5 \pm 3.0 / 25.56 \pm 3.2$ \\
\hline Oh et al. [19] & $100 / 100$ & $69 / 70$ & $54.59 \pm 13.40 / 54.27 \pm 11.52$ & NA:NA/NA:NA & $2.59 \pm 1.35 / 2.52 \pm 1.26$ & $25.14 \pm 2.73 / 25.48 \pm 3.47$ \\
\hline Lee et al. [9] & $234 / 69$ & $164 / 50$ & $54.36 \pm 12.77 / 53.48 \pm 11.85$ & $N A: N A / N A: N A$ & $2.58 \pm 1.40 / 2.37 \pm 1.26$ & $24.49 \pm 2.80 / 25.50 \pm 3.20$ \\
\hline Alemozaffar et al. [7] & $25 / 25$ & $19 / 15$ & $61.9 \pm 10.1 / 55.9 \pm 11.7$ & $0: 25 / 0: 25$ & $3.3 \pm 1.4 / 2.5 \pm 1.0$ & $30.1 \pm 5.9 / 27.5 \pm 3.8$ \\
\hline Simhan et al. 12012 [12] & $136 / 81$ & $89 / 43$ & $58.7 \pm 11.2 / 56.6 \pm 13.1$ & $2: 136 / 3: 81$ & $4.1 \pm 2.3 / 3.2 \pm 1.8$ & $30.0 \pm 7.0 / 30.7 \pm 6.7$ \\
\hline Simhan et al. 22012 [12] & $54 / 10$ & $28 / 6$ & $59.4 \pm 10.8 / 56.1 \pm 10.7$ & 2:54/1:10 & $5.4 \pm 3.8 / 3.7 \pm 2.5$ & $30.9 \pm 6.8 / 30.7 \pm 3.5$ \\
\hline Zargar et al. 12014 [20] & $33 / 30$ & NA/NA & NA/NA & $N A: N A / N A: N A$ & NA/NA & NA/NA \\
\hline Zargar et al. 22014 [20] & $52 / 10$ & NA/NA & NA/NA 7 & NA:NA/NA:NA & NA/NA & NA/NA \\
\hline Boylu et al. [8] & $20 / 46$ & $14 / 29$ & $56 \pm 13.5 / 54 \pm 12$ & $6: 14 / 9: 37$ & $4.04 \pm 2.08 / 3.56 \pm 1.36$ & $27.5 \pm 3.3 / 28.7 \pm 3.1$ \\
\hline Mano et al. [22] & $190 / 63$ & $132 / 46$ & NA/NA & NA:NA/NA:NA & NA/NA & $29(25-31.5) / 29.1(26.6-31.5)^{a}$ \\
\hline Miyake et al. [23] & $15 / 16$ & $10 / 14$ & $64.2 \pm 12.2 / 63.3 \pm 13.2$ & $N A: N A / N A: N A$ & $3.2 \pm 0.9 / 3.0 \pm 0.9$ & $24.4 \pm 5.1 / 24.9 \pm 4.2$ \\
\hline Webb et al. [21] & $21 / 14$ & $14 / 6$ & $53.6 \pm 10.05 / 60.5 \pm 13.45$ & 2:19/2:12 & $4.22 \pm 1.34 / 2.99 \pm 1.10$ & NA/NA \\
\hline
\end{tabular}


Table 3 Perioperative outcomes of RAPN versus OPN

\begin{tabular}{|c|c|c|c|c|c|c|c|c|}
\hline $\begin{array}{l}\text { Study } \\
\text { (OPN/RAPN) }\end{array}$ & $\begin{array}{l}\text { OT } \\
(\min )\end{array}$ & $\begin{array}{l}\text { WIT } \\
(\min )\end{array}$ & $\begin{array}{l}\mathrm{MBL} \\
(\mathrm{ml})\end{array}$ & $\begin{array}{l}\text { Positive } \\
\text { margins (\%) }\end{array}$ & $\begin{array}{l}\text { HS } \\
\text { (day) }\end{array}$ & $\begin{array}{l}\text { eGFR change } \\
\left(\mathrm{ml} / \mathrm{min} \text { per } 1.73 \mathrm{~m}^{2}\right)\end{array}$ & $\begin{array}{l}\text { Transfusion } \\
\text { rate (\%) }\end{array}$ & $\begin{array}{l}\text { Conversion } \\
\text { rate (\%) }\end{array}$ \\
\hline Wu et al. [10] & NA/NA & NA/NA & NA/NA & $\begin{array}{l}0(0.0) / 0 \\
(0.0)\end{array}$ & NA/NA & NA/NA & $4(4.3) / 3(5.9)$ & $\begin{array}{l}1(1.1) / 0 \\
(0.0)\end{array}$ \\
\hline Vittori et al. [11] & $\begin{array}{l}123 \pm 43 / \\
168 \pm 56\end{array}$ & $\begin{array}{l}18.7 \pm 8.1 / \\
18.2 \pm 7\end{array}$ & $\begin{array}{l}230 \pm 208 / \\
125 \pm 128\end{array}$ & $11(5.6) / 6(5.7)$ & NA/NA & NA/NA & NA/NA & $\begin{array}{l}2(1.0) / 1 \\
(0.9)\end{array}$ \\
\hline $\begin{array}{l}\text { Masson-Lecomte } \\
\text { et al. [16] }\end{array}$ & $\begin{array}{l}128.4 \pm 50.5 / \\
134.8 \pm 35.3\end{array}$ & $\begin{array}{l}17.1 \pm 5.9 / \\
17.5 \pm 7.8\end{array}$ & $\begin{array}{l}414.7 \pm 367.5 / \\
142.9 \pm 225.9\end{array}$ & $4(6.9) / 1(2.4)$ & $\begin{array}{l}6.8 \pm 3.5 / \\
3.8 \pm 1.1\end{array}$ & NA/NA & NA/NA & NA/NA \\
\hline Ficarra et al. [17] & NA/NA & NA/NA & NA/NA & $9(4.5) / 9(4.5)$ & NA/NA & $\begin{array}{l}-16.6 \pm 18.1 / \\
-16.4 \pm 22.9\end{array}$ & $\begin{array}{l}20(10.0) / 21 \\
(10.5)\end{array}$ & NA/NA \\
\hline Kim et al. [24] & $\begin{array}{l}126.8 \pm 42.7 / \\
196.9 \pm 50.1\end{array}$ & $\begin{array}{l}27.8 \pm 9.7 / \\
31.3 \pm 9.5\end{array}$ & $\begin{array}{l}356.7 \pm 269.2 / \\
296.8 \pm 246.8\end{array}$ & $1(1.2) / 2(3.0)$ & $\begin{array}{l}4.6 \pm 2.1 / \\
2.9 \pm 1.4\end{array}$ & $\begin{array}{l}-0.49 \pm 17.5 / \\
-2.54 \pm 16.01\end{array}$ & $13(15.7) / 5(7.5)$ & $\begin{array}{l}7(8.4) / 4 \\
(5.9)\end{array}$ \\
\hline Lucas et al. [18] & NA/NA & NA/NA & NA/NA & $4(7.4) / 1(3.7)$ & NA/NA & NA/NA & NA/NA & $\begin{array}{l}0(0.0) / 1 \\
(3.7)\end{array}$ \\
\hline Han et al. [6] & $\begin{array}{l}187.2 \pm 43.8 / \\
162.3 \pm 32.3\end{array}$ & $\begin{array}{l}19.6 \pm 6.7 / \\
24.7 \pm 7.3\end{array}$ & NA/NA & NA/NA & $\begin{array}{l}7.3 \pm 2.06 / \\
5.3 \pm 1.41\end{array}$ & $\begin{array}{l}-4.34 \pm 8.34 / \\
-2.4 \pm 6.7\end{array}$ & NA/NA & NA/NA \\
\hline Oh et al. [19] & $\begin{array}{l}138.79 \pm 40.29 / \\
182.89 \pm 83.98\end{array}$ & $\begin{array}{l}21.18 \pm 11.29 / \\
21.86 \pm 9.25\end{array}$ & $\begin{array}{l}230.74 \pm 159.33 / \\
212.04 \pm 160.76\end{array}$ & $1(1.0) / 0(0.0)$ & $\begin{array}{l}9.26 \pm 3.22 / \\
5.41 \pm 1.84\end{array}$ & $\begin{array}{l}-6.19 \pm 7.32 / \\
-7.53 \pm \\
4.28\end{array}$ & $6(6.0) / 4(4.0)$ & NA/NA \\
\hline Lee et al. [9] & $\begin{array}{l}142.77 \pm 47.69 / \\
192.42 \pm \\
78.05\end{array}$ & $\begin{array}{l}18.14 \pm 7.16 / \\
22.99 \pm 8.43\end{array}$ & $\begin{array}{l}216.50 \pm 165.38 / \\
228.70 \pm 182.89\end{array}$ & $6(2.6) / 0(0.0)$ & $\begin{array}{l}8.90 \pm 3.11 / \\
6.20 \pm 1.99\end{array}$ & $\begin{array}{l}-5.25 \pm 10.01 / \\
-6.11 \pm 9.14\end{array}$ & NA/NA & $\begin{array}{l}1(0.4) / 1 \\
(1.4)\end{array}$ \\
\hline Alemozaffar et al. [7] & $\begin{array}{l}238.3 \pm 119.5 / \\
231.8 \pm 44.2\end{array}$ & NA/NA & $\begin{array}{l}275.4 \pm 170.0 / \\
178.0 \pm 205.7\end{array}$ & NA/NA & $\begin{array}{l}4.60 \pm 1.68 / \\
2.48 \pm 0.68\end{array}$ & NA/NA & NA/NA & NA/NA \\
\hline $\begin{array}{l}\text { Simhan et al. } 1 \\
2012 \text { [12] }\end{array}$ & $\begin{array}{l}189.5 \pm 52.0 / \\
205.9 \pm 52.5\end{array}$ & NA/NA & $\begin{array}{l}256.5 \pm 291.3 / \\
131.3 \pm 127.8\end{array}$ & $1(0.7) / 3(3.7)$ & $\begin{array}{l}5.6 \pm 3.9 / \\
3.7 \pm 1.6\end{array}$ & $\begin{array}{l}1.5 \pm 21 / \\
-2.4 \pm 23.1\end{array}$ & NA/NA & NA/NA \\
\hline $\begin{array}{l}\text { Simhan et al. } 2 \\
2012 \text { [12] }\end{array}$ & $\begin{array}{l}197.5 \pm 60.4 / \\
221.1 \pm 72.5\end{array}$ & NA/NA & $\begin{array}{l}330.6 \pm 406.0 / \\
225.0 \pm 304\end{array}$ & $3(5.6) / 0(0.0)$ & $\begin{array}{l}6.1 \pm 4.1 / \\
2.9 \pm 1.4\end{array}$ & $\begin{array}{l}6.1 \pm 25.2 / \\
-9 \pm 21.2\end{array}$ & NA/NA & NA/NA \\
\hline Zargar et al. 12014 [20] & $\begin{array}{l}185.42 \pm 56.4 / \\
174.9 \pm 61.7\end{array}$ & NA/NA & NA/NA & $3(9.1) / 2(6.7)$ & NA/NA & NA/NA & $5(15.2) / 6(20.0)$ & NA/NA \\
\hline Zargar et al. 22014 [20] & $\begin{array}{l}244.1 \pm 59.3 / \\
250.8 \pm 66\end{array}$ & $\begin{array}{l}23.9 \pm 8.1 / \\
22.7 \pm 5.8\end{array}$ & NA/NA & $4(7.7) / 1(10.0)$ & NA/NA & NA/NA & $8(15.4) / 0(0.0)$ & NA/NA \\
\hline Boylu et al. [8] & $\begin{array}{l}152 \pm 18 / \\
225 \pm 58\end{array}$ & $\begin{array}{l}18 \pm 3.5 / \\
23 \pm 7.3\end{array}$ & $\begin{array}{l}417 \pm 202 / \\
268 \pm 303\end{array}$ & $0(0.0) / 1(2.1)$ & $\begin{array}{l}5.4 \pm 2 / \\
4.11 \pm 1.5\end{array}$ & NA/NA & $2(10.0) / 5(10.9)$ & NA/NA \\
\hline
\end{tabular}


Table 3 Perioperative outcomes of RAPN versus OPN (Continued)

\begin{tabular}{|c|c|c|c|c|c|c|c|c|}
\hline Mano et al. [22] & $\begin{array}{l}128(108-156) / \\
154(113-177)^{b}\end{array}$ & NA/NA & $\begin{array}{l}200(100-413) / \\
100(25-200)^{b}\end{array}$ & NA/NA & $\begin{array}{l}2.47 \pm 1.31 / \\
1.51 \pm 0.76\end{array}$ & NA/NA & NA/NA & NA/NA \\
\hline Miyake et al. [23] & $\begin{array}{l}203.7 \pm 55.2 / \\
263.0 \pm 63.5\end{array}$ & $\begin{array}{l}20.3 \pm 9.1 / \\
23.0 \pm 7.5(1)\end{array}$ & $\begin{array}{l}653.6 \pm 611.7 / \\
57.5 \pm 96.9\end{array}$ & $0(0) / 0(0)$ & $\begin{array}{l}4.8 \pm 0.8 / \\
4.2 \pm 0.8\end{array}$ & $\begin{array}{l}10.0 \pm 6.6 / \\
10.4 \pm 7.0\end{array}$ & $0(0) / 0(0)$ & $\begin{array}{l}0(0) / 0 \\
(0)\end{array}$ \\
\hline Webb et al. [21] & NA/NA & $\begin{array}{l}30.69 \pm 10.65^{a} / \\
28.01 \pm 9.34\end{array}$ & NA/NA & $1(4.8) / 1(7.1)$ & $\begin{array}{l}4(3-6)^{b} / \\
3(2-4)^{b}\end{array}$ & NA/NA & $0(0) / 0(0)$ & $\begin{array}{l}2(9.5) / 0 \\
(0)\end{array}$ \\
\hline
\end{tabular}

The data of OT, WIT, MBL, HS, and eGFR change are expressed as mean \pm standard deviation. The positive margin, transfusion, and conversion are shown with the number of cases and its percentage (the value in brackets) in each study

RAPN robot-assisted partial nephrectomy, OPN open partial nephrectomy; OT operating time; WIT warm ischemia time, MBL mean blood loss, HS hospital stay, eGFR change change of glomerular filtration rate, NA not available

${ }^{\mathrm{b}}$ Median (25th-75th) 


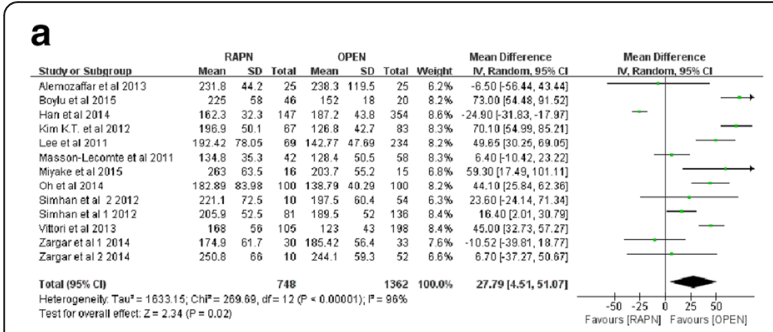

b

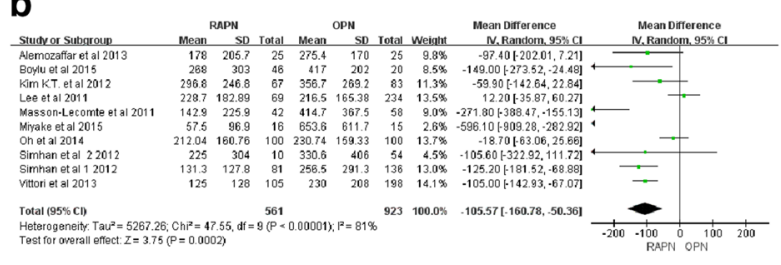

C

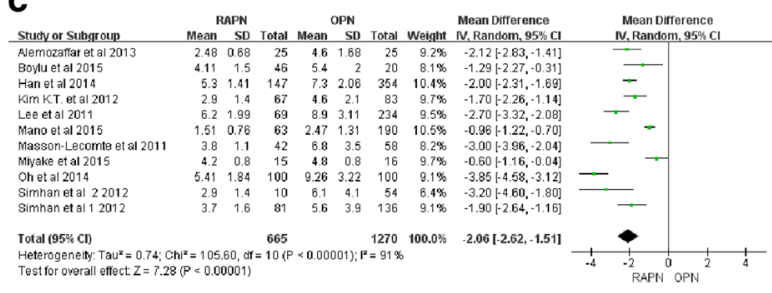

d

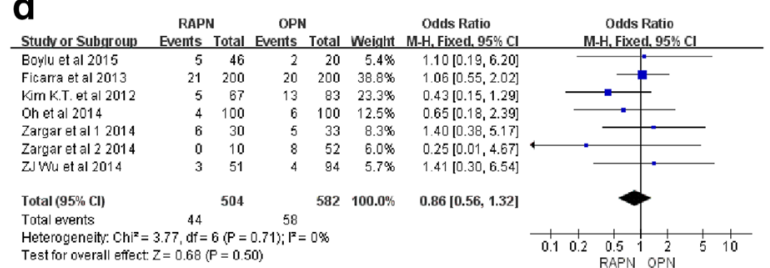

e

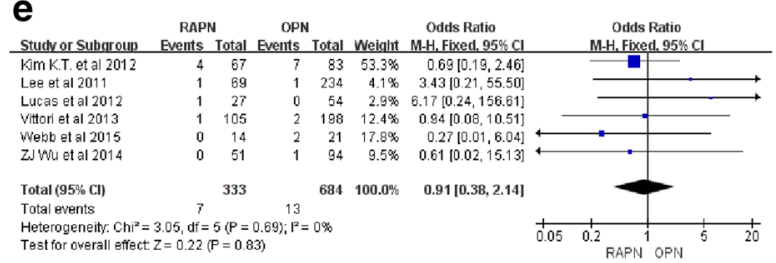

f

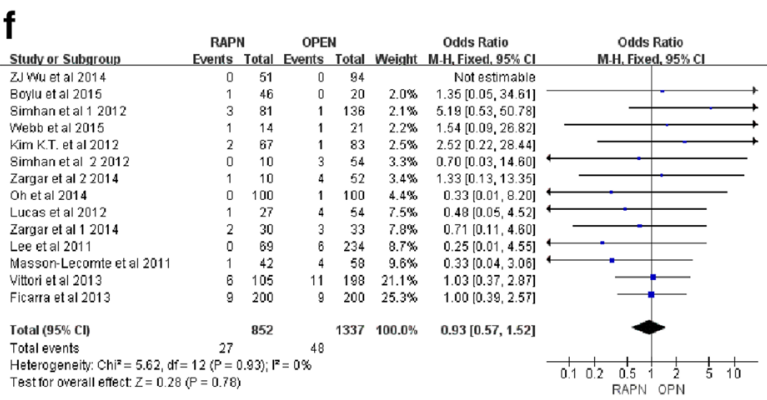

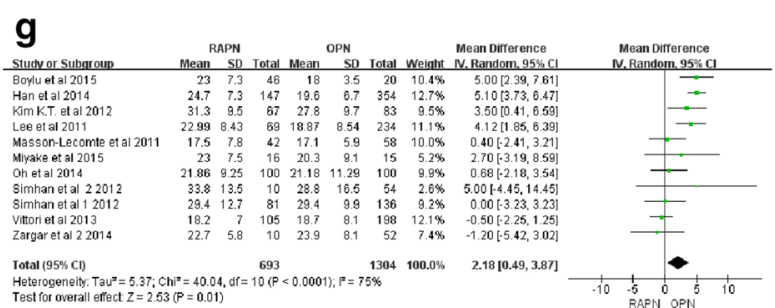

h

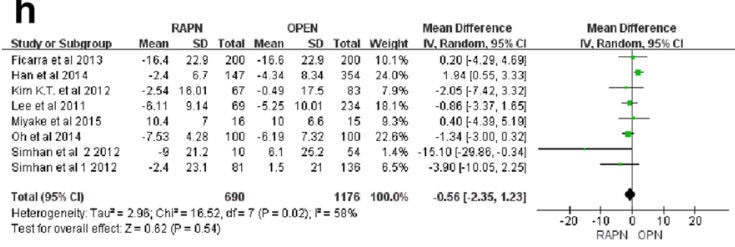

i

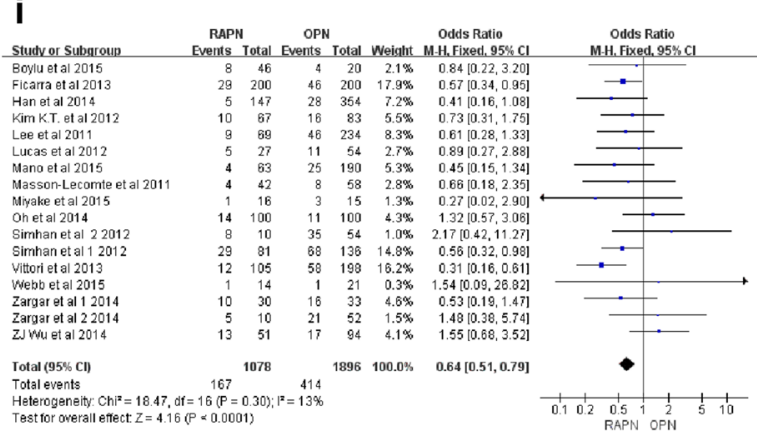

j Studvor Subaroup Events Total Events Total Weight M.-H, Fixed. $95 \%$ Cl

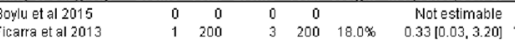

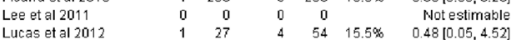

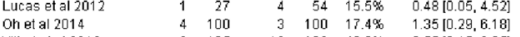

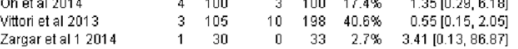

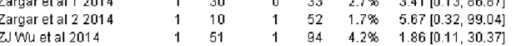
$\begin{array}{llllll}\text { Total }(95 \% \mathrm{Cl}) & 523 & 731 & \mathbf{1 0 0 . 0 \%} & 0.86[0.42,1.76]\end{array}$

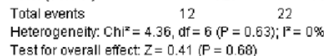

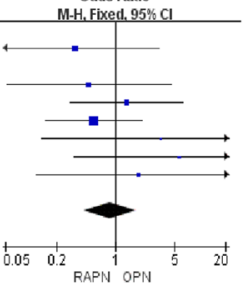

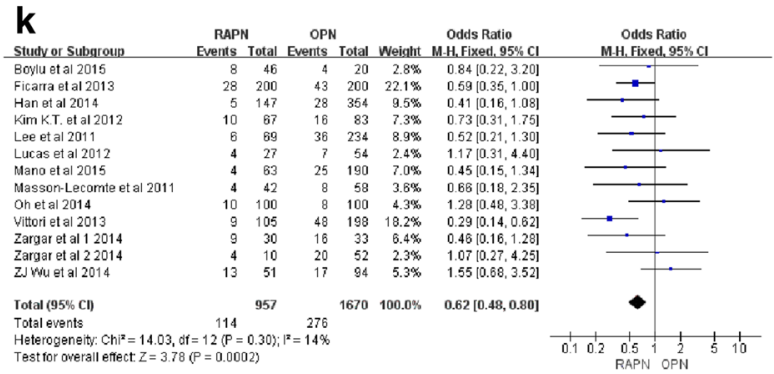

Fig. 2 Forest plots of the perioperative outcomes of RAPN versus OPN. a Operative time. b Estimated blood loss. c Length of hospital stay. $\mathbf{d}$ Transfusion rate. e Rate of conversion to radical nephrectomy. $\mathbf{f}$ Positive margin rate. $\mathbf{g}$ Warm ischemia time. $\mathbf{h}$ Estimated GFR change. i Overall complication rate. $\mathbf{j}$ Intra-operative complicate rate. $\mathbf{k}$ Postoperative complication rate 
Table 4 Complication rates of RAPN versus OPN

\begin{tabular}{|c|c|c|c|c|c|c|c|c|c|c|c|}
\hline $\begin{array}{l}\text { Study } \\
\text { (OPN/RAPN) }\end{array}$ & Patients & $\begin{array}{l}\text { Overall } \\
\text { complication (\%) }\end{array}$ & $\begin{array}{l}\text { Intracomplication } \\
\text { (\%) }\end{array}$ & $\begin{array}{l}\text { Postcomplication } \\
(\%)\end{array}$ & $\begin{array}{l}\text { Clavien1 } \\
(\%)\end{array}$ & $\begin{array}{l}\text { Clavien2 } \\
(\%)\end{array}$ & $\begin{array}{l}\text { Clavien3 } \\
(\%)\end{array}$ & $\begin{array}{l}\text { Clavien4 } \\
\text { (\%) }\end{array}$ & $\begin{array}{l}\text { Clavien5 } \\
(\%)\end{array}$ & $\begin{array}{l}\text { Minor } \\
(\%)\end{array}$ & $\begin{array}{l}\text { Major } \\
(\%)\end{array}$ \\
\hline Wu et al. [10] & $94 / 51$ & $\begin{array}{l}18(19.1) / \\
14(25.9)\end{array}$ & $\begin{array}{l}1(1.1) / \\
1(2.0)\end{array}$ & $\begin{array}{l}17(18.1) / \\
13(25.5)\end{array}$ & \multicolumn{2}{|c|}{$16(17.0) / 12^{a}$} & \multicolumn{3}{|c|}{$1(1.1) / 1(2.0)^{b}$} & $\begin{array}{l}16(17.0) / \\
12(23.5)\end{array}$ & $\begin{array}{l}1(1.1) / \\
1(2.0)\end{array}$ \\
\hline Vittori et al. [11] & $198 / 105$ & $\begin{array}{l}58(29.3) / \\
12(11.4)\end{array}$ & $\begin{array}{l}10(5.1) / \\
3(2.9)\end{array}$ & $\begin{array}{l}48(24.2) / \\
9(8.6)\end{array}$ & $\begin{array}{l}3(1.5) / \\
1(1.1)\end{array}$ & $\begin{array}{l}21(10.6) / \\
4(3.8)\end{array}$ & $\begin{array}{l}15(7.6) / \\
1(1.0)\end{array}$ & $\begin{array}{l}3(1.5) / \\
0(0.0)\end{array}$ & NA/NA & $\begin{array}{l}24(12.1) / \\
5(4.8)\end{array}$ & $\begin{array}{l}18(9.1) / \\
1(1.0)\end{array}$ \\
\hline Masson-Lecomte et al. [16] & $58 / 42$ & $\begin{array}{l}8(13.8) / \\
4(9.6)\end{array}$ & NA/NA & $\begin{array}{l}8(13.8) / \\
4(9.6)\end{array}$ & $\begin{array}{l}0(0.0) / \\
2(4.8)\end{array}$ & $\begin{array}{l}6(10.3) / \\
2(4.8)\end{array}$ & $\begin{array}{l}2(3.4) / \\
0(0.0)\end{array}$ & NA/NA & NA/NA & $\begin{array}{l}6(10.3) / \\
4(9.5)\end{array}$ & $\begin{array}{l}2(3.4) / \\
0(0.0)\end{array}$ \\
\hline Ficarra et al. [17] & $200 / 200$ & $\begin{array}{l}46(23.0) / \\
29(14.5)\end{array}$ & $\begin{array}{l}3(1.5) / \\
1(0.5)\end{array}$ & $\begin{array}{l}43(21.5) / \\
28(14.0)\end{array}$ & $\begin{array}{l}34(17.0) / \\
19(9.5)^{a}\end{array}$ & & $\begin{array}{l}7(3.5) / \\
8(4.0)\end{array}$ & $\begin{array}{l}2(1.0) / \\
1(0.5)\end{array}$ & NA/NA & $\begin{array}{l}34(17.0) / \\
19(9.5)\end{array}$ & $\begin{array}{l}9(4.5) / \\
9(4.5)\end{array}$ \\
\hline Kim et al. [24] & $83 / 67$ & $\begin{array}{l}16(19.3) / \\
10(14.9)\end{array}$ & NA/NA & $\begin{array}{l}16(19.3) / \\
10(14.9)\end{array}$ & $\begin{array}{l}1(1.2) / \\
0(0.0)\end{array}$ & $\begin{array}{l}13(15.7) / \\
7(10.5)\end{array}$ & $\begin{array}{l}0(0.0) / \\
2(3.0)\end{array}$ & $\begin{array}{l}1(1.2) / \\
1(1.5)\end{array}$ & $\begin{array}{l}1(1.2) / \\
0(0)\end{array}$ & $\begin{array}{l}14(16.9) / \\
7(10.4)\end{array}$ & $\begin{array}{l}2(2.4) / \\
3(4.5)\end{array}$ \\
\hline Lucas et al. [18] & $54 / 27$ & $\begin{array}{l}11(20.4) / \\
5(18.5)\end{array}$ & $\begin{array}{l}4(7.4) / \\
1(3.7)\end{array}$ & $\begin{array}{l}7(13.0) / \\
4(14.8)\end{array}$ & $\begin{array}{l}5(9.3) / \\
1(3.7)\end{array}$ & $\begin{array}{l}1(1.9) / \\
2(7.4)\end{array}$ & $\begin{array}{l}1(1.9) / \\
0(0.0)\end{array}$ & $\begin{array}{l}0(0.0) / \\
1(3.7)\end{array}$ & NA/NA & $\begin{array}{l}6(11.1) / \\
3(11.1)\end{array}$ & $\begin{array}{l}1(1.9) / \\
1(3.7)\end{array}$ \\
\hline Han et al. [6] & $354 / 147$ & $\begin{array}{l}28(7.9) / \\
5(3.4)\end{array}$ & NA/NA & $\begin{array}{l}28(7.9) / \\
5(3.4)\end{array}$ & $\begin{array}{l}4(1.1) / \\
2(1.4)\end{array}$ & $\begin{array}{l}17(4.8) / \\
1(0.7)\end{array}$ & $\begin{array}{l}7(2.0) / \\
2(0.2)^{c}\end{array}$ & & NA/NA & $\begin{array}{l}21(5.9) / \\
3(2.0)\end{array}$ & $\begin{array}{l}7(2.0) / \\
2(0.2)\end{array}$ \\
\hline Oh et al. [19] & $100 / 100$ & $\begin{array}{l}11(11.0) / \\
14(14.0)\end{array}$ & $\begin{array}{l}3(3.0) / \\
4(4.0)\end{array}$ & $\begin{array}{l}8(8.0) / \\
10(10.0)\end{array}$ & NA/NA & NA/NA & NA/NA & NA/NA & NA/NA & NA/NA & NA/NA \\
\hline Lee et al. [9] & $234 / 69$ & $\begin{array}{l}46(19.6) / \\
9(13.0)\end{array}$ & $\begin{array}{l}10(4.3) / \\
3(4.3)\end{array}$ & $\begin{array}{l}36(15.4) / \\
6(8.7)\end{array}$ & $\begin{array}{l}20(8.5) / \\
3(4.3)\end{array}$ & $\begin{array}{l}5(2.1) / \\
3(4.3)\end{array}$ & $\begin{array}{l}11(4.7) / \\
0(0.0)\end{array}$ & NA/NA & NA/NA & $\begin{array}{l}25(10.7) / \\
6(8.7)\end{array}$ & $\begin{array}{l}11(4.7) / \\
0(0.0)\end{array}$ \\
\hline Alemozaffar et al. [7] & $25 / 25$ & NA/NA & NA/NA & NA/NA & NA/NA & NA/NA & NA/NA & NA/NA & NA/NA & NA/NA & NA/NA \\
\hline Simhan et al. 12012 [12] & $136 / 81$ & $\begin{array}{l}68(50.0) / \\
29(35.8)\end{array}$ & NA/NA & NA/NA & NA/NA & NA/NA & NA/NA & NA/NA & NA/NA & NA/NA & NA/NA \\
\hline Simhan et al. 22012 [12] & $54 / 10$ & $\begin{array}{l}35(64.8) / \\
8(80.0)\end{array}$ & NA/NA & NA/NA & NA/NA & NA/NA & NA/NA & NA/NA & NA/NA & NA/NA & NA/NA \\
\hline Zargar et al. 12014 [20] & $33 / 30$ & $\begin{array}{l}16(48.5) / \\
10(33.3)\end{array}$ & $\begin{array}{l}0(0.0) / \\
1(3.3)\end{array}$ & $\begin{array}{l}16(48.5) / \\
9(30.0)\end{array}$ & $\begin{array}{l}6(18.2) / \\
3(10)\end{array}$ & $\begin{array}{l}3(9.1) / \\
4(13.3)\end{array}$ & $\begin{array}{l}3(9.1) / \\
2(6.7)\end{array}$ & $\begin{array}{l}4(12.1) / \\
0(0.0)\end{array}$ & NA/NA & $\begin{array}{l}9(27.3) / \\
7(23.3)\end{array}$ & $\begin{array}{l}7(13.5) / \\
2(6.7)\end{array}$ \\
\hline Zargar et al. 22014 [20] & $52 / 10$ & $\begin{array}{l}21(40.4) / \\
5(50.0)\end{array}$ & $\begin{array}{l}1(1.9) / \\
1(10.0)\end{array}$ & $\begin{array}{l}20(38.5) / \\
4(40.0)\end{array}$ & $\begin{array}{l}10(19.2) / \\
0(0)\end{array}$ & $\begin{array}{l}4(7.7) / \\
0(0.0)\end{array}$ & $\begin{array}{l}3(5.8) / \\
1(10.0)\end{array}$ & $\begin{array}{l}3(5.8) / \\
3(30.0)\end{array}$ & NA/NA & $\begin{array}{l}14(26.9) / \\
0(0.0)\end{array}$ & $\begin{array}{l}6(11.5) / \\
4(40.0)\end{array}$ \\
\hline Boylu et al. [8] & $20 / 46$ & $\begin{array}{l}4(20) / \\
8(17.3)\end{array}$ & $\begin{array}{l}0(0.0) / \\
0(0.0)\end{array}$ & $\begin{array}{l}4(20) / \\
8(17.3)\end{array}$ & NA/NA & NA/NA & NA/NA & NA/NA & NA/NA & NA/NA & NA/NA \\
\hline Mano et al. [22] & $190 / 63$ & $\begin{array}{l}25(13) / \\
4(6)\end{array}$ & NA/NA & $\begin{array}{l}25(13) / \\
4(6)\end{array}$ & $\begin{array}{l}14(7) / \\
4(6)^{\mathrm{a}}\end{array}$ & & $\begin{array}{l}11(6) / \\
0(0)\end{array}$ & $\begin{array}{l}0(0.0) / \\
0(0.0)\end{array}$ & $\begin{array}{l}0(0.0) / \\
0(0.0)\end{array}$ & $14(7) / 4(6)$ & $\begin{array}{l}11(6) / \\
0(0)\end{array}$ \\
\hline Miyake et al. [23] & $15 / 16$ & $\begin{array}{l}3(20.0) / \\
1(8.3)\end{array}$ & NA/NA & $\begin{array}{l}3(20.0) / \\
1(8.3)\end{array}$ & $\begin{array}{l}1(6.7) / \\
0(0)^{a}\end{array}$ & & $\begin{array}{l}2(13.3) / \\
1(8.3)^{b}\end{array}$ & & & $\begin{array}{l}1(6.7) / \\
0(0)\end{array}$ & $\begin{array}{l}2(13.3) / \\
1(8.3)\end{array}$ \\
\hline Webb et al. [21] & $21 / 14$ & $\begin{array}{l}1(4.7) / \\
1(7.14)\end{array}$ & NA/NA & NA/NA & NA/NA & NA/NA & NA/NA & NA/NA & NA/NA & NA/NA & NA/NA \\
\hline
\end{tabular}

NA not available

${ }^{\text {aClavien }} 1$ and 2 complication

${ }^{\mathrm{b}}$ Clavien 3-5 complications

CClavien 3 and 4 complications were merged as one group for discussion in the original data sources 

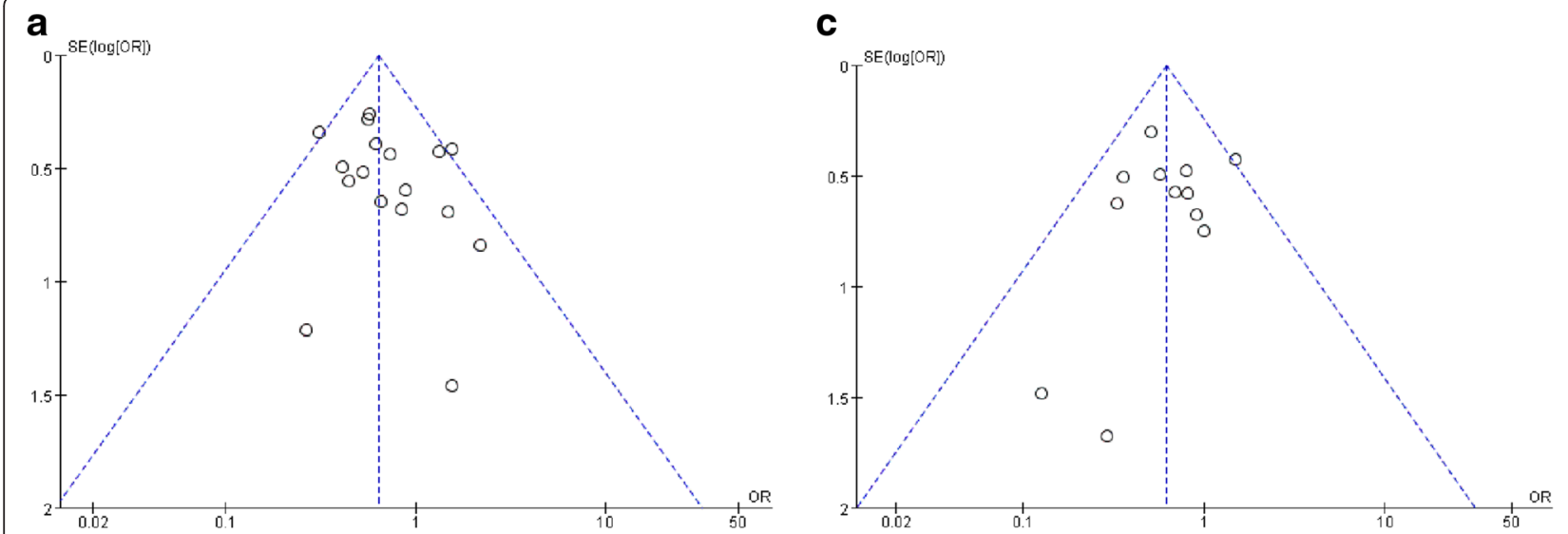

b
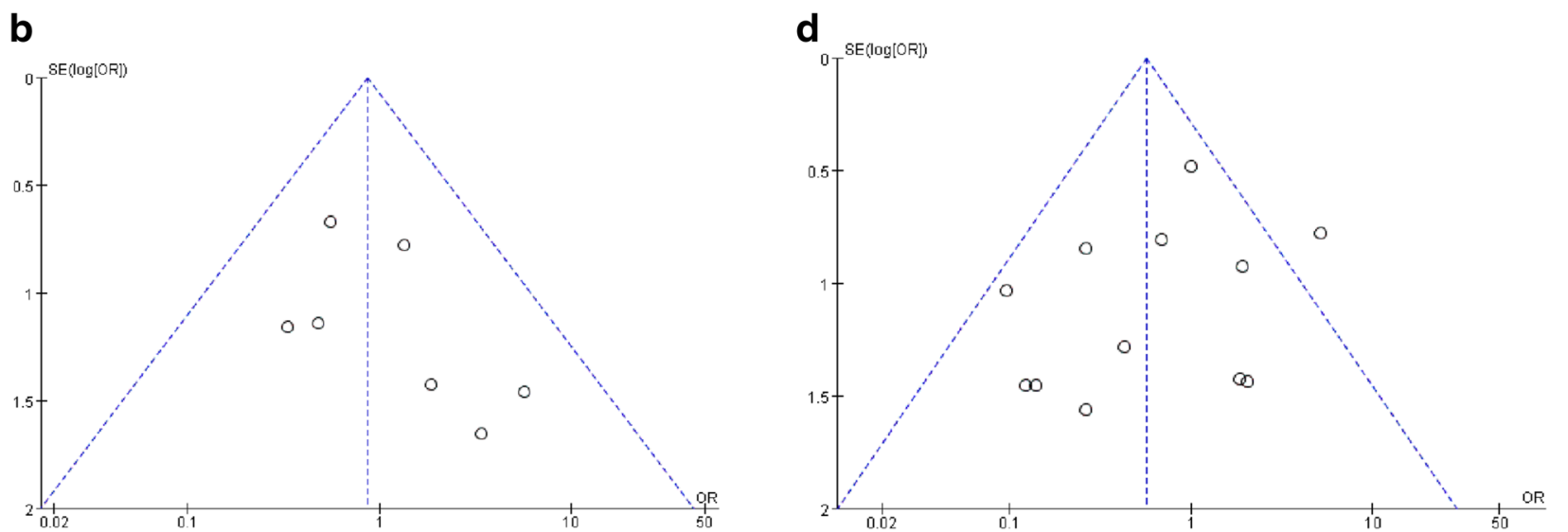

Fig. 3 Funnel plots illustrating meta-analysis of perioperative complication rates. a Overall complication. b Intraoperative complication. c Minor complication. d Major complication. SE standard error, OR odds ratio

and 1921 cases for OPN) to compare the outcomes between RAPN and OPN. We notice that the tumor size of the RAPN group is smaller than that of the OPN group. This could be caused by the selection bias that RAPN is recommended to deal with small renal masses, but we can see that all of the included studies matched the two groups with tumor size except Vittori et al. [11, 22]. Nephrometry score, which derives from the systematic analysis of anatomical renal tumor characteristics, plays an important role in $\mathrm{PN}$ outcomes reporting because it indicates the degree of technical complexity and permits valid comparison among different cohorts. In this meta-analysis, we observed 11 studies $[6,7,11,12,17-23]$ matched the two approaches with renal tumor nephrometry score (RENAL or PADUA). We believe that it is comparable for the two groups. RAPN has a lower age than OPN, but all of the studies included in this meta-analysis matched the two groups with age except Alemozaffar et al. [7]. Together with these, demographic characteristics of the study populations have a little impact on the outcome of the two groups, although the selection bias is existed.

The operative time was significantly longer in the RAPN group than in the OPN group, Masson-Lecomte et al. [16] found that the difference in operative time was insignificant between RAPN and OPN when "skin-to-skin" time (excluding the setup and docking time) rather than the total operating room occupation time. And we can find that the operative time in RAPN group was shorter than Wu et al.'s [10] meta-analysis, we believe that the operative time of RAPN will be shorter than OPN in the future, because RAPA enables a flexible, precise, and rapid operation. On the contrary, RAPN has better outcomes in terms of EBL when compared with the open group. In addition to this, length of hospital stay was significantly shorter in the RAPN group. Boylu et al. [8] found that the mean operation time was significantly longer and the EBL was less for the RAPN group, but they found that the mean postoperative decline of hematocrit was not statistically different between the two groups, which suggests that RAPN provided rapid convalescence, decreased hospital stay, and less blood loss when compared to the OPN group. We did not see the difference in the margin status between the two groups in our analysis either. Additionally, no significant differences in the transfusion rate and surgical conversion rate between RAPN and OPN were observed in this study, which also suggested that RAPN is comparable to OPN. 
Our analysis showed that the overall WIT was higher in the RAPN group. WIT $<30 \mathrm{~min}$ is recommended in order to reduce renal ischemic injury [30], and a more recently published multicenter study suggests that the optimal WIT should be $<20 \mathrm{~min}$ in order to preserve optimal renal functions [5]. Thompson et al. [31] suggest that WIT $<25$ min is a safety standard for partial nephrectomy. Ideal WIT is still under debate in the current literature, but most of the authors agreed that WIT < $25 \mathrm{~min}$ is a safety standard for partial nephrectomy. All of the included studies in this meta-analysis meet the standard, except the Simhan et al. [12] and Kim et al. [24]. Since the amount of renal parenchyma removed rather than WIT is the determinant for the final degree of renal function preservation [32, 33], WIT is unlikely to be the limiting factor for RAPN. Despite the significantly longer WIT in the RAPN group, we found that the change of eGFR is comparable between the two groups, suggesting that RAPN and OPN apparently have the same efficacy on postoperative renal function.

We found that there was no significant difference in intraoperative complication rate between the two groups, which was inconsistent with the finding by Wu et al. [10]. We pooled the data from 7 studies associated with Clavien grade 2 complication, which requires treatment with drugs, and found that RAPN had lower chance of Clavien grade 2 complication than OPN; this can also be seen in Clavien grade 3 complication $(p=0.003)$. Only 1 study compared the Clavien grade 5 complication (death) and found that there was no difference between the two groups $(p=0.421)$. Zhang et al. [34] compared the perioperative and oncologic outcomes of localized renal tumors treated by RAPN with those treated by LPN and found no difference between them. Vittori et al. [11] demonstrated that open surgical approach is the only independent risk factor associated with Clavien grade 3-4 complications. Here, we found that there was no difference between RAPN and OPN regarding Clavien grade 3-4 complications. In the subgroup analysis of postoperative complications, minor complications and major complications frequently occurred following OPN group.

A trend was observed toward a higher failure of cancer control rate for OPN (2.5 versus $0.8 \%$ ), it is not appropriate to estimate the weighted effect with the hazard ratio of tumor recurrence and metastasis for the differences in the length of follow-up duration between the studies.

We realize that there are limitations in this metaanalysis. Firstly, all the included studies are retrospective, non-randomized comparisons, except 2 prospectively derived comparative studies. Secondly, no follow-ups of long period have been achieved for RAPN, so the data availability for tumor recurrence and metastasis were quite limited. Thirdly, all continuous variables had a big heterogeneity which may contribute to the different sample sizes, multiple surgeons with different surgical experiences, tumor complexity, and the lack of RENAL standardization between groups. In addition, almost all previous studies deal with small renal masses. This reminds us a caution of potential selection bias. As a newly emerging surgical option for the treatment of renal masses, RAPN should be compared to OPN that has robust data regarding the surgical and oncological outcomes and serves as the justification standard. Although it will be helpful to perform prospective randomized studies comparing RAPN with OPN, such studies in need of recruiting a homogeneous group of patients with renal masses are difficult to carry out under the context of real clinic.

\section{Conclusions}

This meta-analysis reveals that RAPN results in a significantly lower rate of perioperative complications, less estimated blood loss, and shorter hospital stay, but longer operative time and estimated warm ischemia time than that of open approach. There are no differences in the margin status, transfusion rate, and conversion rate between RAPN and OPN. Thus, RAPN can be an effective alternative to OPN. Well-designed prospective randomized controlled trials will be helpful in validating our findings. With the accumulating knowledge about RAPN, LPN, and OPN, the best decisions regarding the surgical technique for organ-sparing renal tumor resection may be made under the consideration of both patient and surgeon's preference.

\section{Acknowledgements \\ Not applicable.}

\section{Funding}

This project was supported by the Natural Science Foundation of Tianjin (No. 12ZCDZSY16600), the Natural Science Foundation of Tianjin (No. 14JCYBJC26300), the Natural Science Foundation of Tianjin (No. 15JCYBJC24600), and the National Key Specialty Construction of Clinical Projects.

\section{Availability of data and materials}

All data generated or analyzed during this study are included in this published article.

\section{Authors' contributions}

$\mathrm{ZS}, \mathrm{HH}$, and $\mathrm{CW}$ made substantial contributions to the conception and design. TC, HH, LX, CX, and $X L$ are involved in the acquisition of data. $Y Z, Z S$, and ZW helped in the analysis and interpretation of the data; ZS, LX, and WX have been involved in the drafting of the manuscript. DT revised it critically for important intellectual content; CW and DT have given final approval of the version to be published. All authors read and approved the final manuscript.

\section{Competing interests}

The authors declare that they have no competing interests.

\section{Consent for publication}

Not applicable.

Ethics approval and consent to participate Not applicable. 


\section{Author details}

'Department of Urology, The Second Hospital of Tianjin Medical University, Pingjiang Road 23Hexi District, Tianjin 300211, China. ${ }^{2}$ Tianjin Key Laboratory of Urology, Tianjin Institute of Urology, the Second Hospital of Tianjin Medical University, Tianjin 300211, China. ${ }^{3}$ Key Laboratory of Genetics and Birth Health of Hunan Province, The Family Planning Research Institute of Hunan Province, Changsha, Hunan 410126, China.

Received: 16 December 2015 Accepted: 3 August 2016

Published online: 22 August 2016

\section{References}

1. Torre LA, Bray F, Siegel RL, Ferlay J, Lortet-Tieulent J, Jemal A. Global cancer statistics, 2012. CA: A Cancer Journal for Clinicians. 2015;65(2):87-108.

2. Ljungberg B, Bensalah $\mathrm{K}$, Canfield $\mathrm{S}$, et al. EAU guidelines on renal cell carcinoma: 2014 update. Eur Urol. 2015;67(5):913-924.

3. Thompson RH, Boorjian SA, Lohse CM, et al. Radical nephrectomy for pT1a renal masses may be associated with decreased overall survival compared with partial nephrectomy. The Journal of Urology. 2008;179(2):468-73.

4. Uzzo RG, Novick AC. Nephron sparing surgery for renal tumors: indications, techniques and outcomes. J Urol. 2001;166(1):6-18.

5. Ficarra $\mathrm{V}$, Bhayani $\mathrm{S}$, Porter J, et al. Predictors of warm ischemia time and perioperative complications in a multicenter, international series of robotassisted partial nephrectomy. European Urology. 2012:61(2):395-402.

6. Han KS, Song GH, You D, et al. Comparison of hand-assisted laparoscopic versus robot-assisted laparoscopic versus open partial nephrectomy in patients with T1 renal masses. J Endourol. 2014. doi:10.1089/end.2014.0517.

7. Alemozaffar M, Chang SL, Kacker R, Sun M, DeWolf WC, Wagner AA Comparing costs of robotic, laparoscopic, and open partial nephrectomy. J Endourol. 2013:27(5):560-5.

8. Boylu U, Basatac C, Yildirim U, Onol F, Gumus E. Comparison of surgical, functional, and oncological outcomes of open and robot-assisted partial nephrectomy. Journal of Minimal Access Surgery. 2015;11(1):72.

9. Lee S, Oh J, Hong SK, Lee SE, Byun SS. Open versus robot-assisted partial nephrectomy: effect on clinical outcome. J Endourol. 2011;25(7):1181-5.

10. Wu Z, Li M, Qu L, et al. A propensity-score matched comparison of perioperative and early renal functional outcomes of robotic versus open partial nephrectomy. PLoS One. 2014;9(4), e94195.

11. Vittori G. Open versus robotic-assisted partial nephrectomy: a multicenter comparison study of perioperative results and complications. World Journal of Urology. 2014;32(1):287-93.

12. Simhan J, Smaldone MC, Tsai KJ, et al. Perioperative outcomes of robotic and open partial nephrectomy for moderately and highly complex renal lesions. J Urol. 2012;187(6):2000-4.

13. Higgins JP, Thompson SG, Deeks JJ, Altman DG. Measuring inconsistency in meta-analyses. BMJ. 2003;327(7414):557-60.

14. Taggart DP, D'Amico R, Altman DG. Effect of arterial revascularisation on survival: a systematic review of studies comparing bilateral and single internal mammary arteries. Lancet. 2001;358(9285):870-5.

15. Dindo D, Demartines N, Clavien PA. Classification of surgical complications: a new proposal with evaluation in a cohort of 6336 patients and results of a survey. Annals of Surgery. 2004;240(2):205-13.

16. Masson-Lecomte A, Yates DR, Hupertan V, et al. A prospective comparison of the pathologic and surgical outcomes obtained after elective treatment of renal cell carcinoma by open or robot-assisted partial nephrectomy. Urol Oncol. 2013:31:924-9.

17. Ficarra $V$, Minervini A, Antonelli A, et al. A multicentre matched-pair analysis comparing robot-assisted versus open partial nephrectomy. BJU International. 2014;113(6):936-41.

18. Lucas SM, Mellon MJ, Erntsberger L, Sundaram CP. A comparison of robotic laparoscopic and open partial nephrectomy. JSLS. 2012;16(4):581-7.

19. Oh JJ, Byun S, Hong SK, Jeong CW, Lee SE. Comparison of robotic and open partial nephrectomy: single-surgeon matched cohort study. Can Urol Assoc J. 2014:8(7-8):E471-5.

20. Zargar H, Bhayani S, Allaf ME, et al. Comparison of perioperative outcomes of robot-assisted partial nephrectomy and open partial nephrectomy in patients with a solitary kidney. Journal of Endourology. 2014;28(10):1224-30.

21. Webb CM, Kamel M, Eltahawy E, et al. A comparative study of open, laparoscopic and robotic partial nephrectomy in obese patients. Urol Ann. 2015;7(2):231-4.
22. Mano R, Schulman A, Hakimi AA, et al. Cost comparison of open and robotic partial nephrectomy using a short postoperative pathway. Urology. 2015;85(3):596-603.

23. Miyake H, Hinata N, Imai S, Furukawa J, Tanaka K, Fujisawa M. Partial nephrectomy for hilar tumors: comparison of conventional open and robotassisted approaches. International Journal of Clinical Oncology. 2015;20(4):808-13.

24. Kim KT, Choo MS, Lee HE, et al. Comparison of robot-assisted partial nephrectomy and open partial nephrectomy: clinical outcome and complication analysis. Europena urology supplements. 2012;11(1):E32-U352.

25. Gill IS, Kavoussi LR, Lane BR, et al. Comparison of 1,800 laparoscopic and open partial nephrectomies for single renal tumors. J Urol. 2007;178(1):41-6.

26. Porpiglia F, Volpe A, Billia M, Scarpa RM. Laparoscopic versus open partial nephrectomy: analysis of the current literature. European Urology. 2008:53(4):732-42. 742-743.

27. Rogers CG, Patard JJ. Open to debate. The motion: robotic partial nephrectomy is better than open partial nephrectomy. European Urology. 2009;56(3):568-70.

28. Laydner $\mathrm{H}$, Isac W, Autorino R, et al. Single institutional cost analysis of 325 robotic, laparoscopic, and open partial nephrectomies. Urology. 2013;81(3):533-9.

29. Yu H, Hevelone ND, Lipsitz SR, Kowalczyk KJ, Hu JC. Use, costs and comparative effectiveness of robotic assisted, laparoscopic and open urological surgery. The Journal of Urology. 2012;187(4):1392-9.

30. Novick AC. Renal hypothermia: in vivo and ex vivo. Urol Clin North Am. 1983:10(4):637-44

31. Thompson $\mathrm{RH}$, Lane BR, Lohse $\mathrm{CM}$, et al. Renal function after partial nephrectomy: effect of warm ischemia relative to quantity and quality of preserved kidney. Urology. 2012;79(2):356-60.

32. Mir MC, Campbell RA, Sharma N, et al. Parenchymal volume preservation and ischemia during partial nephrectomy: functional and volumetric analysis. Urology. 2013;82(2):263-8.

33. Simmons MN, Hillyer SP, Lee BH, Fergany AF, Kaouk J, Campbell SC. Functional recovery after partial nephrectomy: effects of volume loss and ischemic injury. J Urol. 2012;187(5):1667-73.

34. Zhang X, Yan J, Ren Y, Shen C, Ying X, Pan S. Robot-assisted versus laparoscopic partial nephrectomy for localized renal tumors: a meta-analysis. International Journal of Clinical and Experimental Medicine. 2014:7(12):4770-9.

\section{Submit your next manuscript to BioMed Central and we will help you at every step:}

- We accept pre-submission inquiries

- Our selector tool helps you to find the most relevant journal

- We provide round the clock customer support

- Convenient online submission

- Thorough peer review

- Inclusion in PubMed and all major indexing services

- Maximum visibility for your research

Submit your manuscript at www.biomedcentral.com/submit 